\title{
Josephson Current in a Ferromagnetic Semiconductor/Semiconductor/Ferromagnetic Semiconductor Junction with Superconducting Contacts
}

\author{
Y.C. Tao \\ Department of Physics, Nanjing Normal University, Nanjing \\ People's Republic of China
}

\section{Introduction}

Superconducting spintronics [1-3] is one of the most attractive subjects of spintronics. A variety of spin-injecting tunnelling junctions composed of ferromagnet and superconductor (SC) with different wave pairing symmetries have been explored theoretically and experimentally, where the ferromagnet includes ferromagnetic metals (FM), ferromagnetic semiconductors (FS), and so on.

Recently, the FS arouses great interests due to several important material properties, for example, carrier type (i.e., $n$ or $p$ ), carrier densities, and ferromagnetism, can be easily controlled in it. [4] The origin of ferromagnetism in the FS can be explained by applying a picture that uniform itinerant carrier spin mediates a long-range ferromagnetic order between the ions $\mathrm{Mn}^{+2}$ with spin. $p$-type III-V GaMnAs is a prototype FS with a large spin polarization even for small Mn concentrations [4,5] and recent experiments demonstrate that the Curie temperature of this material can be as high as $150 \mathrm{~K},[4,5]$ indicating promise for possible technological relevance. [5-8]

On the other hand, current can flow without dissipation through a thin insulating layer or weak link separating two superconductors (SC). A phase difference $\phi$ between the SCs appears on account of the quantum character of the superflow and the current-phase relationship of the link is periodic function of $\phi$. For a tunnel barrier, it is given by $I=$ $I_{C} \sin (\phi)$ as first found by Josephson [9], where $I_{C}$ is the critical Josephson current. When the two SCs coupled by a thin ferromagnetic layer [10-29], $I_{C}$ can change sign. Referring to the Josephson current-phase relationship, the change is corresponding to a $\pi$-phase shift of $\phi$. And thus Josephson junctions can present a negative coupling, which are usually called $\pi$ junctions. The transitions from positive to negative coupling (the so-called transition from 0 to $\pi$ junction) by increasing the FS layer thickness observed by experiments are qualitatively in agreement with those of theoretical calculations. It has been widely accepted that high-critical

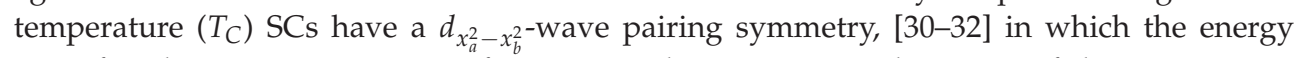
gap of such a pairing state manifests a sign change at some directions of the Fermi wave 
vector. This feature is much different from that for a conventional s-wave SC. As a result of this sign change, it was predicted [33] that a sizable areal density of midgap states exists on a $\{110\}$ surface of a $d$-wave SC. These midgap states have exerted the influence on the Josephson current of high $T_{C}$ SC junctions. More recently, the relative Josephson tunnelling junctions composed of FS and SC with s-wave pairing symmetry have being explored and some valuable results have been obtained, $[34,35]$ however, the works focused on the systems are scarce. Particularly, to our knowledge, we seldom see theoretical and experimental works taking into account the unconventional pairing symmetry, which is relevant to the high $T_{C}$ superconducting junctions composed of the FS. Therefore, it seems to be timely to review the Superconducting Spintronics based on the Josephson junctions consisting of FS and SC with s-wave pairing symmetry and consider the outlook for the future work.

In this chapter, the Josephson current flowing through an FS/semiconductor (SM) /FS trilayer connected to two superconductor (SC) electrodes is studied. We extend a general expression for dc Josephson current to the structure, in which the quasiparticle wave function, satisfying Bogoliubov-de Gennes (BDG) equation, is first obtained and then the temperature Green's function formalism is used. The Josephson current in the junction is find to be strongly dependent on not only the kinds of holes in the FS's and scattering potential strengths at the interfaces but also the relative orientations of the effective exchange field of the two FS's. We also show that in the coherent regime, the oscillatory dependences of the critical Josephson current on the FS and SM layer thicknesses and Josephson current on the macroscopic phase difference for the heavy and light holes in different orientations of the effective exchange field of the two FS's, are much different due to the different mismatches in the effective mass and Fermi velocity between the FS and SC, which is connected with the transition from positive (0) to negative $(\pi)$ coupling. The effect can be exploited for the design of devices such as superconducting quantum interference devices with improved accuracy and convenience. In Sec. II, we present the theoretical approach. In Sec. III, FS Josephson tunneling junctions are studied. The summary and outlook are given in Sec. IV. Finally, the list of used symbols is presented in Sec. V.

\section{Theoretical approach: BDG equation}

Applying Bogoliubov-de Gennes (BDG) equation, [36] Superconducting Spintronics built on the Josephson junctions composed of GaMnAs FS and s-wave SC are studied, in which the $\mathrm{AR},[34,35]$ hole types, mismatches in the effective mass and Fermi velocity of holes between the FS and SC, and strengths of potential scattering at the interface are taken into account. In the next parts of the chapter, the layers are taken to be the $y-z$ plane and to be stacked along the $x$-direction, and the FS/SC interface, for example at $x=a$, is described by a $\delta$-type potential $V(x)=U \delta(x-a)$ with $U$ dependent on the product of the barrier height and width.

As in FMs, a spin-polarized subband model with the spins parallel and antiparallel to the local magnetization is extended to the heavy and light hole bands. The hole Hamiltonian in the FS is simply given by

$$
H_{F S}(\boldsymbol{r})=H_{0}(\boldsymbol{r})-\boldsymbol{h}(\boldsymbol{r}) \cdot \boldsymbol{\sigma} .
$$

Here $H_{0}(\boldsymbol{r})=-\hbar^{2} \nabla_{\boldsymbol{r}} / 2 m_{p}+V(\boldsymbol{r})$ is the kinetic energy with $m_{p}$ the mass of a hole plus the usual static potential, which is also the hole Hamiltonian of the SM GaAs, $\boldsymbol{h}(\boldsymbol{r})$ is the effective exchange field with the magnitude equal to $\Gamma / 2$, and $\sigma$ the conventional Pauli spin operator. 
At finite temperatures, the spin splitting energy $\Gamma$ different for different kind of holes, is given by $\Gamma=J_{p d}<S^{z}>$, where $J_{p d}$ stands for the $p-d$ exchange coupling strength between itinerant holes and $\mathrm{Mn}^{2+}$ ion impurity spins connected with the densities of Mn impurity and heavy or light holes, and $\left\langle S^{z}>\right.$ implies the thermal average of the impurity Mn ion spins. To model the subband spin splitting and the relative spin-polarized degree $P$, we define the parameter $P \equiv \Gamma / E_{F}$. The SC is assumed s-wave pairing and described by a BCS-like Hamiltonian, in which the excitation energy is $\zeta_{k}=\sqrt{\epsilon_{k}^{2}+\Delta^{2}}$ with $\epsilon_{k}=\hbar^{2} k^{2} / 2 m_{e}-E_{F}$ the one-electron energy relative to $E_{F}$ and $\Delta$ the energy gap. The energy gap $\Delta$ is determined by the self-consistent equation $[34,35]$

$$
\ln \left(\frac{\Delta_{0}}{\Delta}\right)=\int_{0}^{\hbar \omega_{D}} \frac{d \epsilon_{k}}{\zeta_{k}} \frac{2}{1+e^{\beta \zeta_{k}}}
$$

where $\omega_{D}$ refers to Debye frequency and $\Delta_{0}$ denotes energy gap at zero temperature.

The quasiparticle wave functions, in the Blonder-Tinkham-Klapwijk model, BDG equation [36]

$$
\left[\begin{array}{cc}
H_{0}^{S C}(\boldsymbol{r})-\eta_{\sigma} h(\boldsymbol{r}) & \Delta(x) \\
\Delta^{*}(x) & -H_{0}^{S C^{*}}(\boldsymbol{r})+\eta_{\bar{\sigma}} h(\boldsymbol{r})
\end{array}\right]\left[\begin{array}{c}
u_{\sigma} \\
v_{\bar{\sigma}}
\end{array}\right]=E\left[\begin{array}{c}
u_{\sigma} \\
v_{\bar{\sigma}}
\end{array}\right]
$$

where $u_{\sigma}$ and $v_{\bar{\sigma}}$ are the two-component wavefunction, $H_{0}^{S C}(\boldsymbol{r})$ has the same expression as $H_{0}^{F S}(\boldsymbol{r})$ except for the effective mass replaced by the mass of electron $m_{e}, \Delta(x)$ is pair potential, $E$ is the quasiparticle energy relative to $E_{F}, \bar{\sigma}$ is the spin opposite to $\sigma$ with $\uparrow$ and $\downarrow, \eta_{\sigma}=1$ for $\sigma=\uparrow$, and $\eta_{\sigma}=-1$ for $\sigma=\downarrow$. In Eq. (3), as a result of that the spin-flip process has been neglected in the SC near interfaces in Refs. 34 and 35, the spin-dependent (four-component) BDG equation may be decoupled into two sets of two-component equations: $u_{\sigma}, v_{\bar{\sigma}}$ describing the spin- $\sigma$ electronlike quasiparticle (ELQ) and spin- $\bar{\sigma}$ holelike quasiparticle (HLQ), respectively.

\section{FS Josephson tunnelling junctions}

\subsection{Model and theory}

A SC/FS/SM/FS/SC Josephson tunnel junction as in Fig. 1 is considered [34, 35]. The left and right electrodes are made up of the same SCs: they are separated from a FS/SM/FS trilayer with the same FS GaMnAs layers of thickness $b$ and SM GaAs of thickness $a$. Either the left and right FS/SC interfaces or SM/FS interfaces, can be approximately described by four $\delta$-type barrier potentials $V(x)=U \delta[z \pm(b+a / 2)]$ and $V^{\prime}(x)=U^{\prime} \delta(z \pm a / 2)$, respectively and assumed to be symmetrical due to the same SCs and the same FS's, where $U$ and $U^{\prime}$ depend on the product of the barrier height and width. For the ferromagnetic (F) alignment of the magnetizations of the two bilayers, $\boldsymbol{h}(-b-a / 2 \leq z \leq-a / 2)=\boldsymbol{h}(a / 2 \leq z \leq b+a / 2)$, while for the antiferromagnetic (A) alignment, $\boldsymbol{h}(-b-a / 2 \leq z \leq-a / 2)=-\boldsymbol{h}(a / 2 \leq z \leq b+a / 2)$. For the injection of an ELQ from the left SC with Andreev reflection as a HLQ $(a)$ and normal reflection $(b)$, the wave function are given by $[34,35]$

$$
\psi_{L}^{S C}(x)=\left[e^{i k^{+} x}+b e^{-i k^{+} x}\right]\left(\begin{array}{l}
u e^{i \phi_{L} / 2} \\
v e^{-i \phi_{L} / 2}
\end{array}\right)+a e^{i k^{-} x}\left(\begin{array}{l}
v e^{i \phi_{L} / 2} \\
u e^{-i \phi_{L} / 2}
\end{array}\right),
$$




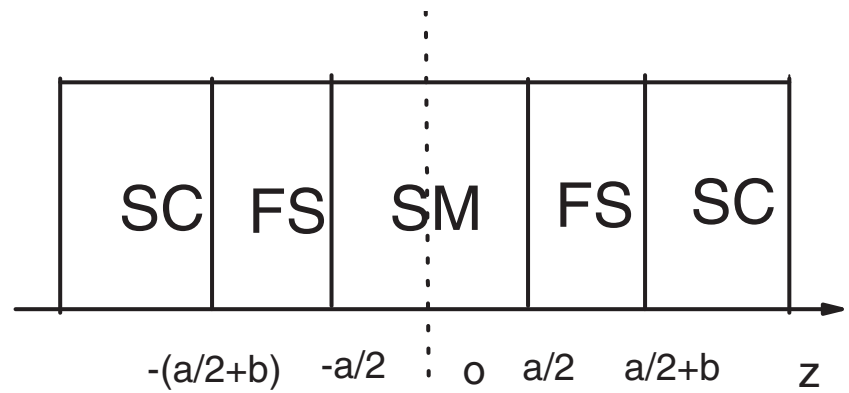

Fig. 1. The SC/FS/SM/FS/SC system (from Ref. 34).

for $x \leq-b-a / 2$,

$$
\psi_{1}(x)=\left[c_{11} e^{i q_{\sigma}^{L+} x}+c_{12} e^{-i q_{\sigma}^{L+} x}\right]\left(\begin{array}{l}
1 \\
0
\end{array}\right)+\left[c_{13} e^{i q_{\bar{\sigma}}^{L-} x}+c_{14} e^{-i q_{\bar{\sigma}}^{L-} x}\right]\left(\begin{array}{l}
0 \\
1
\end{array}\right)
$$

for $-b-a / 2 \leq x \leq-a / 2$,

$$
\psi_{2}(z)=\left[c_{21} e^{i q^{\prime+} x}+c_{22} e^{-i q^{\prime+} x}\right]\left(\begin{array}{l}
1 \\
0
\end{array}\right)+\left[c_{23} e^{i q^{\prime-} x}+c_{24} e^{-i q^{\prime-} x}\right]\left(\begin{array}{l}
0 \\
1
\end{array}\right)
$$

for $-a / 2 \leq x \leq a / 2$

$$
\psi_{3}(x)=\left[c_{31} e^{i q_{\sigma}^{R+} x}+c_{32} e^{-i q_{\sigma}^{R+} x}\right]\left(\begin{array}{l}
1 \\
0
\end{array}\right)+\left[c_{33} e^{i q_{\bar{\sigma}}^{R-} x}+c_{34} e^{-i q^{R-} \bar{\sigma}^{x}}\right]\left(\begin{array}{l}
0 \\
1
\end{array}\right)
$$

for $a / 2 \leq x \leq a / 2+b$,

$$
\psi_{R}^{S C}(x)=c e^{i k^{+} x}\left(\begin{array}{l}
u e^{i \phi_{R} / 2} \\
v e^{-i \phi_{R} / 2}
\end{array}\right)+d e^{-i k^{-} x}\left(\begin{array}{l}
v e^{i \phi_{R} / 2} \\
u e^{-i \phi_{R} / 2}
\end{array}\right),
$$

for $x \geq a / 2+b$, where $q_{\sigma}^{L(R) \pm}=\sqrt{\left(2 m_{p} / \hbar^{2}\right)\left(E_{F} \pm E+\eta_{\sigma} h_{0}\right)-k_{\|}^{2}}, q^{\prime \pm}=$ $\sqrt{\left(2 m_{p} / \hbar^{2}\right)\left(E_{F} \pm E\right)-k_{\|}^{2}}, k^{ \pm}=\sqrt{\left(2 m_{e} / \hbar^{2}\right)\left(E_{F} \pm \Omega\right)-k_{\|}^{2}}, u=\sqrt{(1+\Omega / E) / 2}$, and $v=$ $\sqrt{(1-\Omega / E) / 2}$ with $\Omega=\sqrt{E^{2}-\Delta^{2}}$ and the parallel component of the wave vector $k_{\|}$ which is conserved. In Eqs. (4)-(8), all coefficients will be determined by the usual matching conditions of the wave functions.

From Eqs. (4)-(8), the transmission and reflection coefficients can be obtained. [34, 35] In the derivation, two dimensionless parameters $Z=2 m_{e} U /\left(\hbar^{2} k_{F}^{S C}\right)$ and $Z^{\prime}=2 m_{e} U^{\prime} /\left(\hbar^{2} k_{F}^{S C}\right)$, and $\chi$ are respectively introduced to describe the barrier strengths at the interfaces and ratio of the masses $m_{p}$ and $m_{e}$, the phase difference between the SCs $\phi=\phi_{L}-\phi_{R},\left|k_{\|}\right|=k_{F}^{S C} \sin \theta$, where the corresponding Fermi vector in the SC is $k_{F}^{S C}=\sqrt{2 m_{e} E_{F}} / \hbar$. Analogously, one can easily obtain the Andreev reflection $\left(a^{\prime}\right)$ as an ELQ for the injection of a HLQ into the right FS from the left SC.

After having obtained the coefficients $a$ and $a^{\prime}$, we can express the dc Josephson current in terms of the Andreev reflections amplitudes by using the temperature Green's function 
formalism [37]

$$
I=\frac{e \Delta}{2 \hbar} \sum_{\sigma, k_{\|}} k_{B} T \sum_{\omega_{n}} \frac{1}{2 \Omega_{n}}\left(k_{n}^{+}+k_{n}^{-}\right)\left(\frac{a_{n}}{k_{n}^{+}}-\frac{a_{n}^{\prime}}{k_{n}^{-}}\right),
$$

where $k_{n}^{+}, k_{n}^{-}, a_{n}$, and $a_{n}^{\prime}$ are obtained from $k^{+}, k^{-}, a$, and $a^{\prime}$ by the analytic continuation $E \rightarrow i \omega_{n}$, the Matsubara frequencies are $\omega_{n}=\pi k_{B} T(2 n+1)$ with $n=0, \pm 1, \pm 2, \ldots$, and $\Omega_{n}=\sqrt{\omega_{n}^{2}+\Delta^{2}}$. As for the same $E, \sigma$, and $\theta$ in the approximation with $\Omega / E_{F} \ll 1$ and $E / E_{F} \ll 1$, the amplitudes of two Andreev reflections $a(\phi)$ and $a^{\prime}(\phi)$ are simply connected by $a^{\prime}(\phi)=a(-\phi)$. Performing integration over $k_{\|}$, we can deduce [34, 35]

$$
I=\frac{2 \pi k_{B} T \Delta}{e R k_{F}^{S C}} \int_{0}^{\pi / 2} d \theta \sin \theta \cos \theta \sum_{\omega_{n}, \sigma}\left[a_{n}(\phi)-a_{n}(-\phi)\right],
$$

where $R=2 \pi^{2} \hbar / S e^{2}\left(k_{F}^{S C}\right)^{2}$ with $S$ the area of junction. In accordance with Eq. (10), we can calculate the Josephson currents in the $\mathrm{F}$ and A alignments.

\subsection{Calculations and results}

In Fig. 2 are illustrated the numerical results for $I_{C}$ as a function of the FS thickness $b$ with $a=40 / k_{F}^{S C}$ at different scattering potential strengths $Z$ and $Z^{\prime}$ for the heavy and light holes in the $\mathrm{F}$ and A alignments. [34] The damped oscillations of the critical Josephson currents $I_{C}$ for the heavy and light holes are all found to be as a function of $b$, which is also the characteristic feature of the ballistic SC/FM/SC junctions and always related to the crossovers between 0 and $\pi$ states [24]. Microscopically, Andreev reflections play an important role in the transfer of Cooper pairs through the FS. An electronlike quasiparticle in FS with energy lower than the superconducting energy gap $\Delta$, cannot enter into the SC, but is reflected at the FS/SC interface as a hole and reflected back as an electron at the opposite SC/FS interface. The constructive interference of the electronlike and holelike excitations gives rise to Andreev bound states in FS, which carry the supercurrent. However, in the FS, the spin splitting of spin band exerts an influence on the Andreev reflection when the spin of the quasiparticles is reversed by the Andreev reflection, which means that the exchange energy is gained or lost by a quasiparticle Andreev-reflected at the FS/SC interface. It follows that the superconducting pair amplitude induced in FS by the proximity to the SC is spatially modulated and the phase increases linearly with the distance from the SC/FS interface. Since the Josephson critical current $I_{C}$ is proportional to the pair amplitude in the FS, $I_{C}$ follows the sign of the order parameter in the FS. Whether it is either positive or negative is determined by the phase accumulating in the FS. Furthermore, it is found that as shown in Fig. 2, at $Z=0$ and $Z^{\prime}=0$, for the heavy holes, the general tendency of variations of the oscillation amplitude with increasing $b$ is to first decrease, then increase, and last gradually reduce to zero in the F and A alignments, while for the light holes, gradually decrease to zero all the time. We also find that for either the heavy or light holes, the behaviors in the $\mathrm{F}$ and $\mathrm{A}$ alignments are very similar in spite of the values of $Z$ and $Z^{\prime}$. However, for $Z$ and $Z^{\prime}$ unequal to zero, it is noticeable that the peak values for heavy holes all have bigger difference between the $\mathrm{F}$ and $\mathrm{A}$ alignments, whereas for the light holes, no much bigger difference, which is the same as that for $Z=0$ and $Z^{\prime}=0$. Besides, with the increase of the barrier strengths $Z$ and $Z^{\prime}$, the peak values all decrease for the two kinds of holes in the F and A alignments, however, the number of periodicity is decreased for 


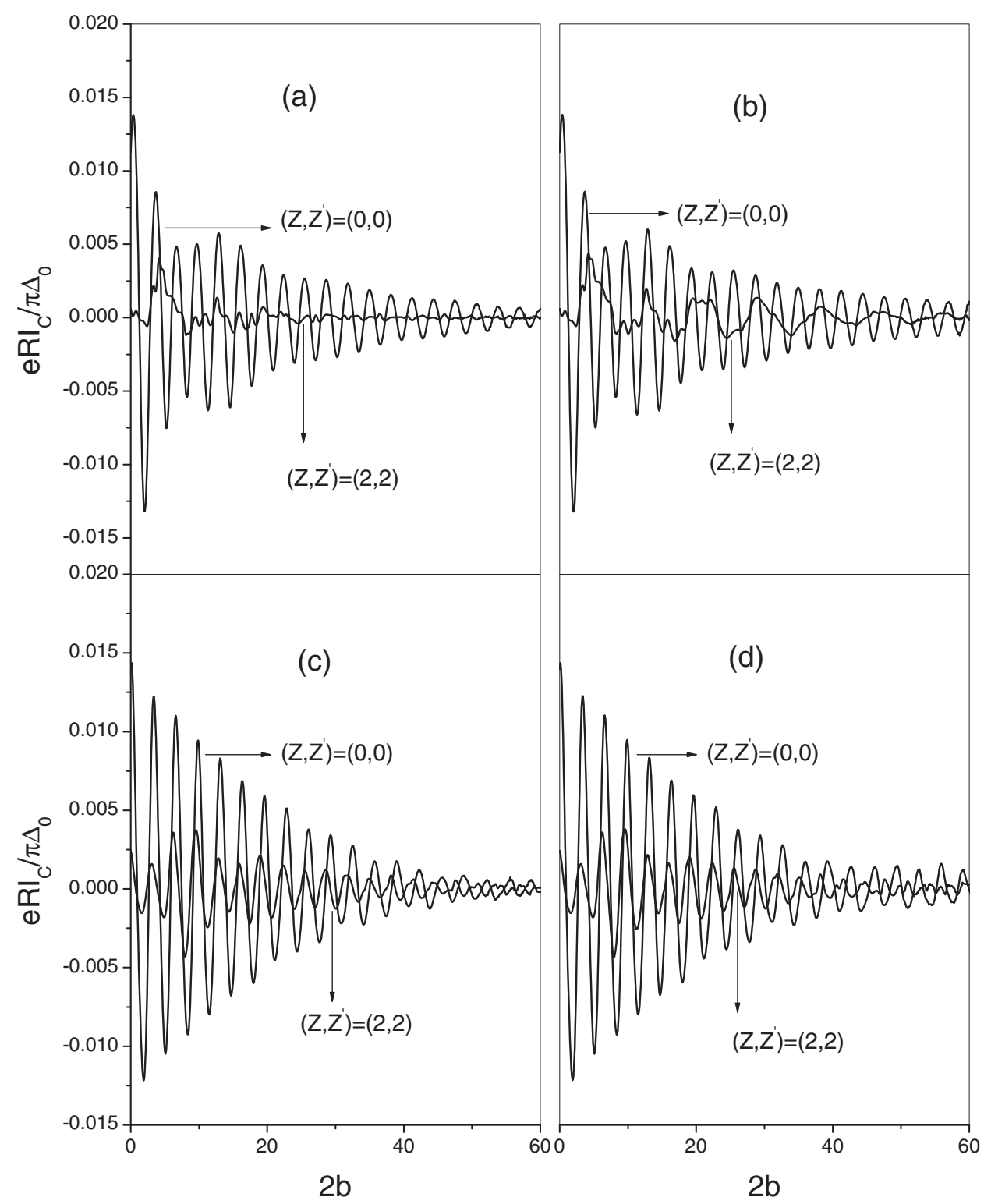

Fig. 2. Dependences of $I_{C}$ on the FS thickness $b$ at different interface scattering potential strengths $\left(Z, Z^{\prime}\right)$ with $(0,0)$ (solid line) and $(2,2)$ (dotted line). Here, $E_{F}=100.0 \mathrm{meV}$, $\Delta_{0}=1.4 \mathrm{meV}, k_{B} T=0.05 \Delta_{0}, m_{e}=1.0, m_{p}=0.45 m_{e}$ for the heavy holes in the $\mathrm{F}(\mathrm{a})$ and $\mathrm{A}(\mathrm{b})$ alignments, and $m_{p}=0.08$ for the light holes in the F (c) and A (d) (from Ref. 34).

the heavy holes while that for the light is increased. The occurrence of periodic behavior can be explained by the two terms $\exp ^{-i\left[\left(k^{ \pm} \pm q^{\prime \pm}\right) a / 2+\left(k^{ \pm} \pm q_{\sigma}^{L \pm}\right) b-\phi\right]}$ in Eqs. (A4)-(A13) of Appendix 
A, [34] including the product of $b$ and wave vector $q^{L(R)_{ \pm}}$and varying periodically. However, the Fermi vector $q_{F}$ for the heavy holes is much bigger than that for the light because of effective mass bigger than that for the light, as a result, the number of the periodicity for the heavy is also slightly bigger than that for the light as shown in Fig. 2 at $Z=0$ and $Z^{\prime}=0$. More importantly, when $b$ is smaller, $\exp ^{-i\left[\left(k^{ \pm} \pm q^{\prime \pm}\right) a / 2\right.}$ have the bigger effect on the peak value for the heavy holes than that for the light holes, so the behavior of the periodicity for the two holes are different at the smaller $b$. In fact, the effect of the coefficients of the above two terms corresponds to a physical phase for each equation, therefore, when $Z$ and $Z^{\prime}$ are unequal to zero and vary, the phase shift appears and the peaks all shift with the $b$. The phase shifts for heavy and light holes are different on account of different mismatches in the Fermi velocity between the FS and SC, resultantly, the behaviors for the variations of the number of the periodicity are different with $Z$ and $Z^{\prime}$. For the effect of $Z$ and $Z^{\prime}$ on the critical Josephson current, physically, for the heavy or light holes, the exchange energy is not easily or lost by a quasiparticle Andreev-reflected at the the FS/SC interface with bigger $z$, therefore, Josephson current can not easily pass the junction and thus decreases with increasing the $Z$ and $Z^{\prime}$.

Fig. 3 shows the SM thickness $a$ dependence of Josephson critical current $I_{C}$ for the heavy and light holes in the $F$ and $A$ alignments at different $Z$ and $Z^{\prime}$. [34] We find that there are same features as in Fig. 2 except that the behaviors and values of the critical Josephson currents $I_{C}$ are slightly different, which can be similarly easily interpreted as in Fig. 2.

In Figs. 4 (5) are illustrated the phases of the Josephson current $I(\phi)$ of the heavy (light) holes for different $Z$ and $Z^{\prime}$ in the F and A alignments. [34] It is shown that, for the dependence of the Josephson currents on the phase, if the junction is the 0 or $\pi$ state or transition from the 0 to $\pi$ state is determined by not only the magnetic alignment but also kinds of the carriers in the FS. No matter what $P, Z$ and $Z^{\prime}$ are, for the heavy hole, the junctions in the $\mathrm{F}$ and $\mathrm{A}$ alignments are only the 0 state, otherwise these are $\pi$ state, while for the light holes, the situations are thoroughly different from those for the heavy. In the F alignment, regardless of $P$, the minimum values of $Z$ and $Z^{\prime}$ at the same time when there is a transition of the junction changing from the 0 to $\pi$ state are respectively 1.5 and 1.7 or so although the transition is not too obvious for the bigger $P$. If one of $Z$ and $Z^{\prime}$ is increased, the other is at least the above value and $P$ is only decreased for the existence of the transition. In the A alignment, ii spite of $P$, the minimum values of $Z$ and $Z^{\prime}$ at the same time when there exists a transition of the junction changing from the 0 to $\pi$ state are basically the same as those in the F. However, in the A alignment, with the increase of $Z, Z^{\prime}$ can be decreased in small magnitude and $P$ is only enhanced for the existence of the transition, yet both of $Z$ and $Z^{\prime}$ can be increased in the meantime and can be bigger for the bigger $P$. At the half metallic limit, they are respectively approximate to 2.2 and 2.1. It can be concluded from these results that the different mismatches in the effective mass and Fermi velocity between the FS and SC and the relative orientations of the effective exchange field of the two FS's play an important role in transition of the junction changing from the 0 to $\pi$ state. The interpretation can be given as follows. In Eqs. (A4)-(A7) of Appendix A, [34] there are two terms, including $\exp ^{-i\left[\left(K^{ \pm}-q_{\sigma}^{L \pm}\right) b-\phi\right]}$ and $\exp ^{-i\left[\left(K^{ \pm}+q_{\sigma}^{L \pm}\right) b-\phi\right]}$. If the junction is the 0 or $\pi$ state or transition from the 0 to $\pi$ state is mainly dependent on the contribution of the two terms. For the heavy holes, due to the effective mass bigger than that in the SC, Fermi vectors in the F and A alignments are not much smaller compared with those in the SC, therefore the sum of the two terms can not easily change the sign with $\phi$ increased, therefore, there only 0 or $\pi$ state. 


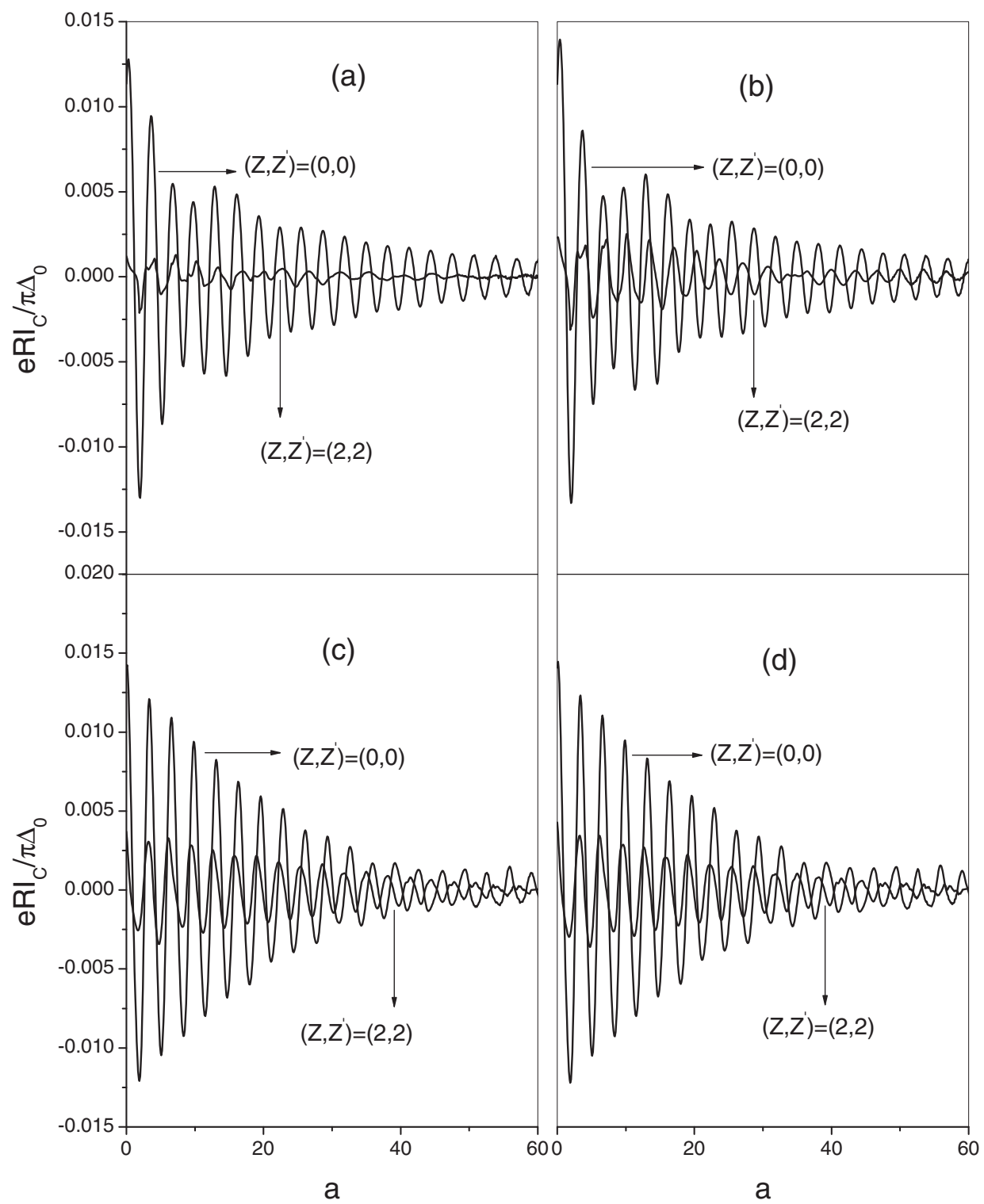

Fig. 3. Dependences of $I_{C}$ on the SM thickness $a$ at $b=20 / k_{F}^{S C}$ with the same situations as in Fig. 2 (from Ref. 34).

However, as concerned about the light holes, for the $\mathrm{F}$ alignment in which the spin of the majority (minority) holes is still that of the majority (minority) holes in the right FS, since the effective mass is much smaller than that for the heavy hole, the Fermi vector is much smaller 
than those for the heavy and in the SC, and thus $\exp ^{-i\left[\left(K^{ \pm}-q_{\sigma}^{L \pm}\right) b-\phi\right]}$ and $\exp ^{-i\left[\left(K^{ \pm}+q_{\sigma}^{L \pm}\right) b-\phi\right]}$ can easily change the sign, particularly for the minority holes. It follows that the general tendency is that smaller $P$ more easily gives rise to the sign variation of the sum of the two terms. In the A alignment, a majority (minority) spin in the right FS will be regard as a minority (majority) spin in the right FS, therefore the increase of $P$ is favorable for the sign change of the sum of the two terms. Furthermore, the barrier strengths in coefficients of the two terms in Eqs. (A4)-(A7) also exert an important influence on the sign of the value. When the two barrier strengths $Z$ and $Z^{\prime}$ or one of them is much bigger or smaller, one of the two coefficients is zero, as result, the increase of $\phi$ can not cause the change of sign of the sum.

In Fig. 6, the normalized critical currents $I_{C}$ at different scattering potential strengths $Z$ and $Z^{\prime}$ in the $\mathrm{F}$ alignment for the heavy and light holes are shown as a function of $P^{\prime}$ [35], where $P^{\prime}$ is define as $P^{\prime} \equiv \Gamma / \Delta_{0}=P E_{F} / \Delta_{0}$ [28]. It is found that the critical Josephson current $I_{C}$ for the heavy holes, at $Z=0$ and $Z^{\prime}=0$, increases with $P^{\prime}$, while for the light, decreases. For $Z$ or $Z^{\prime}$ unequal to zero, the situations are very different. As $Z^{\prime}=0$, for the heavy hole, $I_{C}$ always increases with $P^{\prime}$ at the small $Z$ and decreases at the big $Z$, however, as $Z=0$, at some $Z^{\prime}, I_{C}$ increases with $P^{\prime}$, and at other $Z^{\prime}$, decreases. For the light hole, the critical Josephson currents $I_{C}$ are found, at $Z^{\prime}=0$, to decrease all the time with $P^{\prime}$ regardless of $Z$, while at $Z=0$, there are the same features of $I_{C}$ with variation of $P^{\prime}$ as those for heavy holes.

In Fig. 7, are illustrated the $P^{\prime}$ dependences of Josephson critical current $I_{C}$ for the heavy and light holes in the A alignment at different $Z$ and $Z^{\prime}$. [35] It is shown that there exist some features similar to those in Fig. 6, for example, as $Z\left(Z^{\prime}\right)=0$, for the heavy hole, $I_{C}$ increases at some $Z^{\prime}$ with $P^{\prime}$ and decreases at other $Z^{\prime}$. Simultaneously, it is also found that some behaviors and values of the critical Josephson currents $I_{C}$ with $P^{\prime}$ increased are remarkably different from those in Fig. 6, which are obviously due to the existence of spin splitting energy in the FS's and resultant different mismatches in the Fermi vectors between the FS and SC for the $\mathrm{F}$ and $\mathrm{A}$ alignments. The critical Josephson current in the $\mathrm{A}$ alignment $I_{C}$ for the heavy holes, at $Z=0$ and $Z^{\prime}=0$, decreases with $P^{\prime}$, while for the light, basically has no change. In addition, $I_{C}$ in the A alignment, at $Z\left(Z^{\prime}\right)=0$, always increases with $P^{\prime}$ for the light holes regardless of $Z^{\prime}(Z)$.

Fig. 8 shows the $Z\left(Z^{\prime}\right)$ dependence of Josephson critical current $I_{C}$ for different $Z^{\prime}(Z)$ in the F alignment for the heavy and light holes. [35] The critical Josephson currents $I_{C}$ for the heavy holes, is found to exhibit oscillations with $Z\left(Z^{\prime}\right)$ enhanced, finally reduce to zero and there are two peaks, however, at $Z^{\prime}=0$, firstly increase with the increase of $Z$, whereas at $Z=0$, firstly decreases and then increases with increasing $Z^{\prime}$. For the light hole, the critical Josephson currents $I_{C}$ is also found to display oscillations with $Z\left(Z^{\prime}\right)$ increased. In the meantime, we find that as $Z^{\prime}=0, I_{C}$ firstly decreases with the enhancement of $Z^{\prime}$, while as $Z=0, I_{C}$ firstly increases and then decreases with the increase of $Z^{\prime}$. At $Z$ and $Z^{\prime}$ unequal to zero, the varied behaviors of $I_{C}$ with $Z^{\prime}$ and $Z$ are also very similar, and $I_{C}$ basically decreases, then increases, and lastly decreases to zero with increasing $Z^{\prime}$ or $Z$, which indicates that there is only a peak.

In Fig. 9, the variations of Josephson critical current $I_{C}$ for the heavy and light holes with $Z\left(Z^{\prime}\right)$ at different $Z^{\prime}(Z)$ in the A alignment are plotted. [35]It is found that although there are the same features as those in Fig. 8 , the values of the critical Josephson currents $I_{C}$ with increasing $Z$ or $Z^{\prime}$ are a little different, this can be also ascribed to the same reason as in the explanation of the same and different properties between Figs. 6 and 7. Here, the differences 

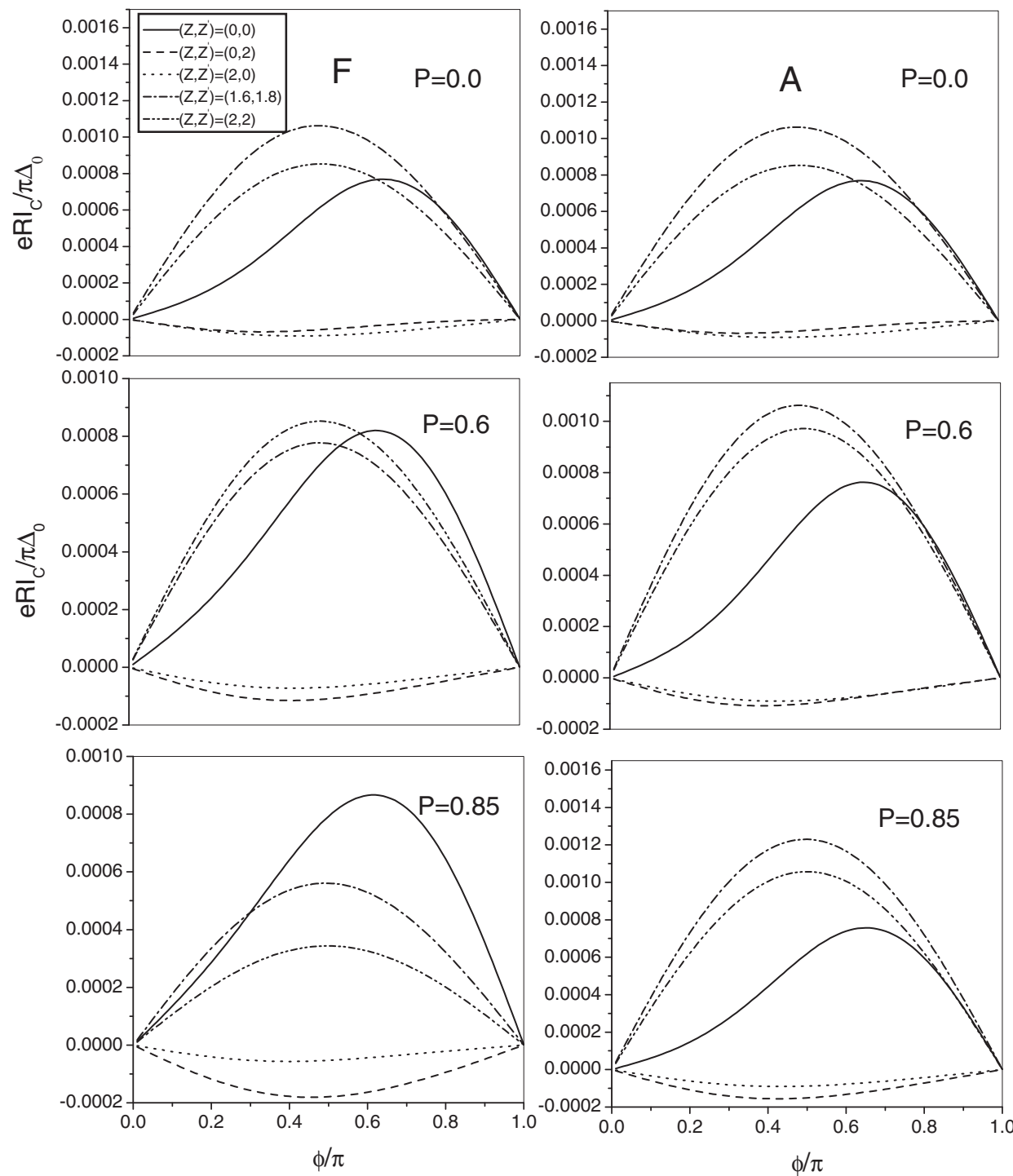

Fig. 4. The phases of the Josephson current $I(\phi)$ for the heavy holes at different $\left(Z, Z^{\prime}\right)$ in the $\mathrm{F}$ and A alignments. Here, $a=40 / k_{F}^{S C}, b=20 / k_{F}^{S C}, Z$ and $Z^{\prime}$ are given in the inset of Fig. 4, and the other parameters are the same as in Fig. 2 (from Ref. 34).

of the results between the heavy and light holes in Fig. 9, together with Figs. 6-8, can be all owing to the fact that there are the different mismatches in the effective mass and Fermi velocity between the FS and SC for the two kinds of holes. 


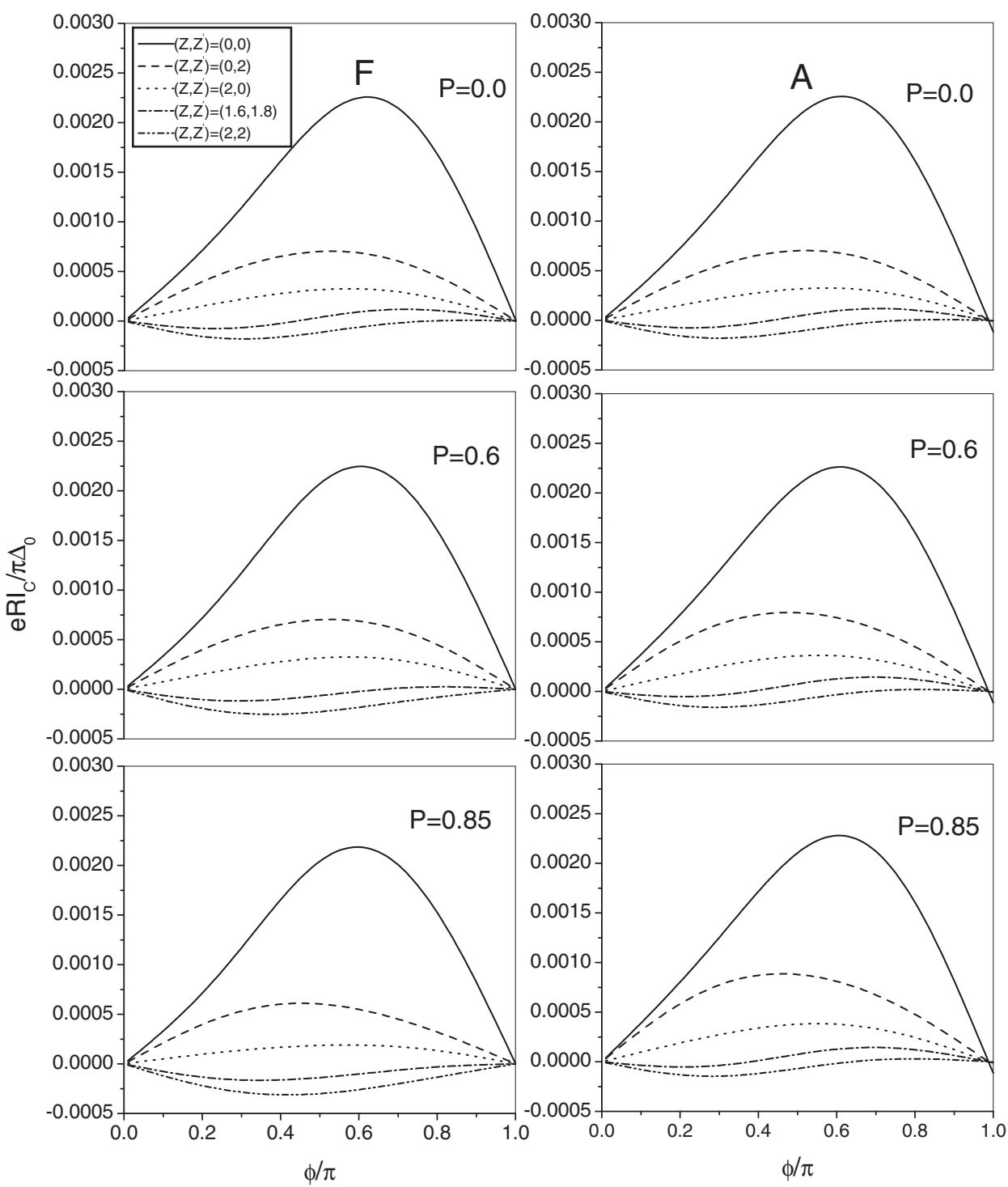

Fig. 5. The same dependence for the light holes as in Fig. 4 (from Ref. 34).

Last, I want to briefly mention that if at finite voltage, there also can exist the dc current which exhibits the so-called subharmonic structure [38,39], i.e., current singularities at voltages $V=$ $2 \Delta /$ en with $n=, 12, \ldots$, where $\Delta$ the energy gap in the SC. Therefore, in such situation, the decoherence due to the subgap currents would have to be taken into account as pointed by Ref. [39]. 


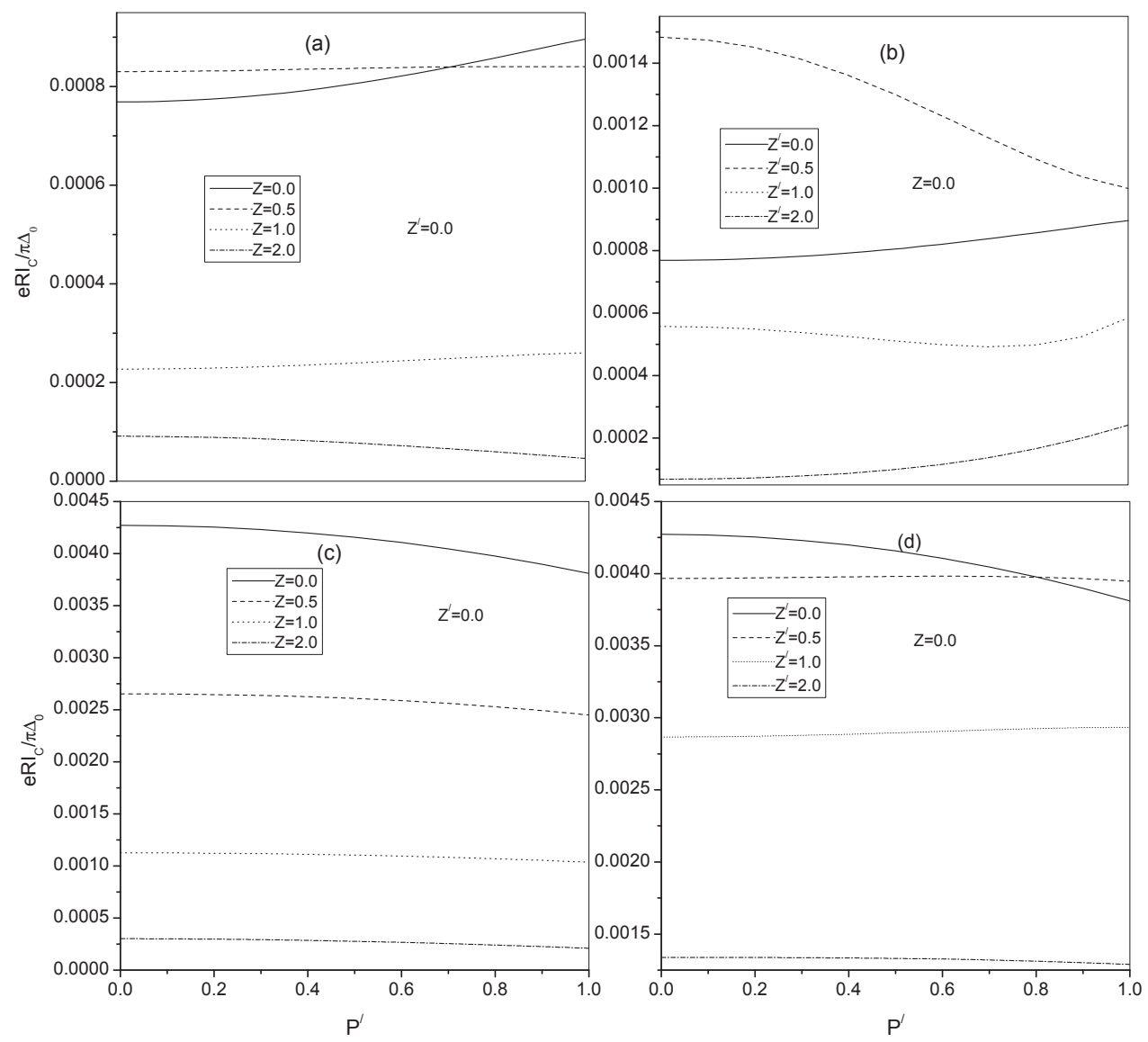

Fig. 6. Dependence of the normalized critical current $I_{C}$ on $P$ in the $\mathrm{F}$ alignment at different scattering potential strengths: $Z$ (a) and $Z^{\prime}$ (b) for the heavy holes; $Z$ (c) and $Z^{\prime}$ (d) for for the light. Here. the parameters are the same as in Fig. 4 (from Ref. 35).

\section{Summary and outlook}

In this chapter, we study the Josephson current flowing through an FS/semiconductor (SM) /FS trilayer connected to two superconductor (SC) electrodes. It is shown that the Josephson current in the junction is strongly dependent on not only the kinds of holes in the FS's and scattering potential strengths at the interfaces but also the relative orientations of the effective exchange field of the two FS's. We also find that in the coherent regime, the oscillatory dependences of the critical Josephson current on the FS and SM layer thicknesses and Josephson current on the macroscopic phase difference for the heavy and light holes in different orientations of the effective exchange field of the two FS's, are much different due to the different mismatches in the effective mass and Fermi velocity between the FS and SC, 


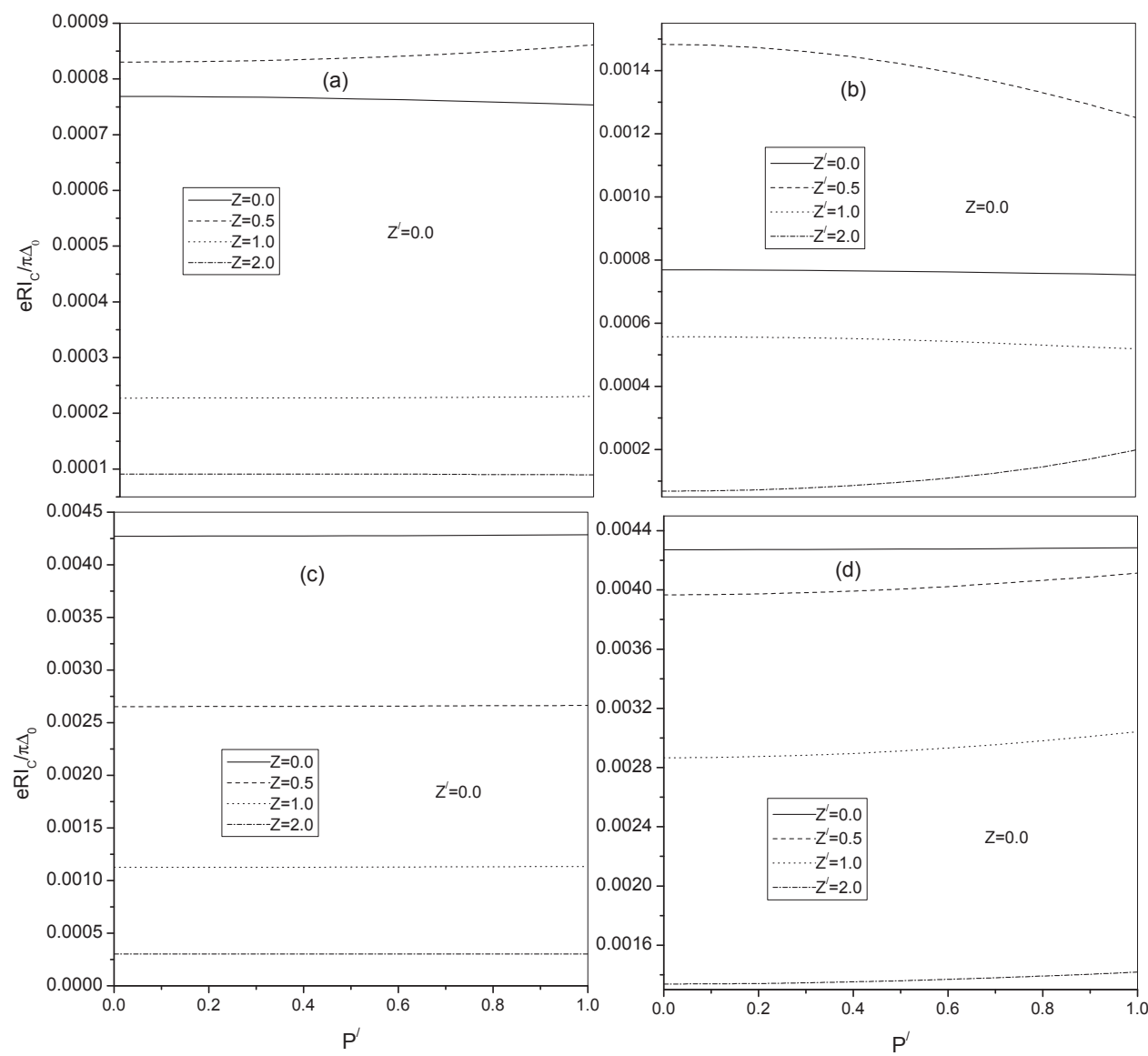

Fig. 7. The same dependence as in Fig. 6 in the case of the A alignment (from Ref. 35).

which is connected with the transition from positive (0) to negative $(\pi)$ coupling. The effect can be exploited for the design of devices such as superconducting quantum interference devices with improved accuracy and convenience.

Up now, we hardly find theoretical and experimental works taking into account the unconventional pairing symmetry, which is relevant to the high $T_{C}$ superconducting junctions comprising the FS. Due to the interplay between the various pairing symmetry in the unconventional SC and hole types in the FS, there will exhibit substantial physical content, which will have not only great intrinsic scientific importance but also potential device applications in the future. We will focus our attention on the superconducting Spintronics based on the junctions consisting of FS and SC with unconventional pairing symmetry in the future work. 

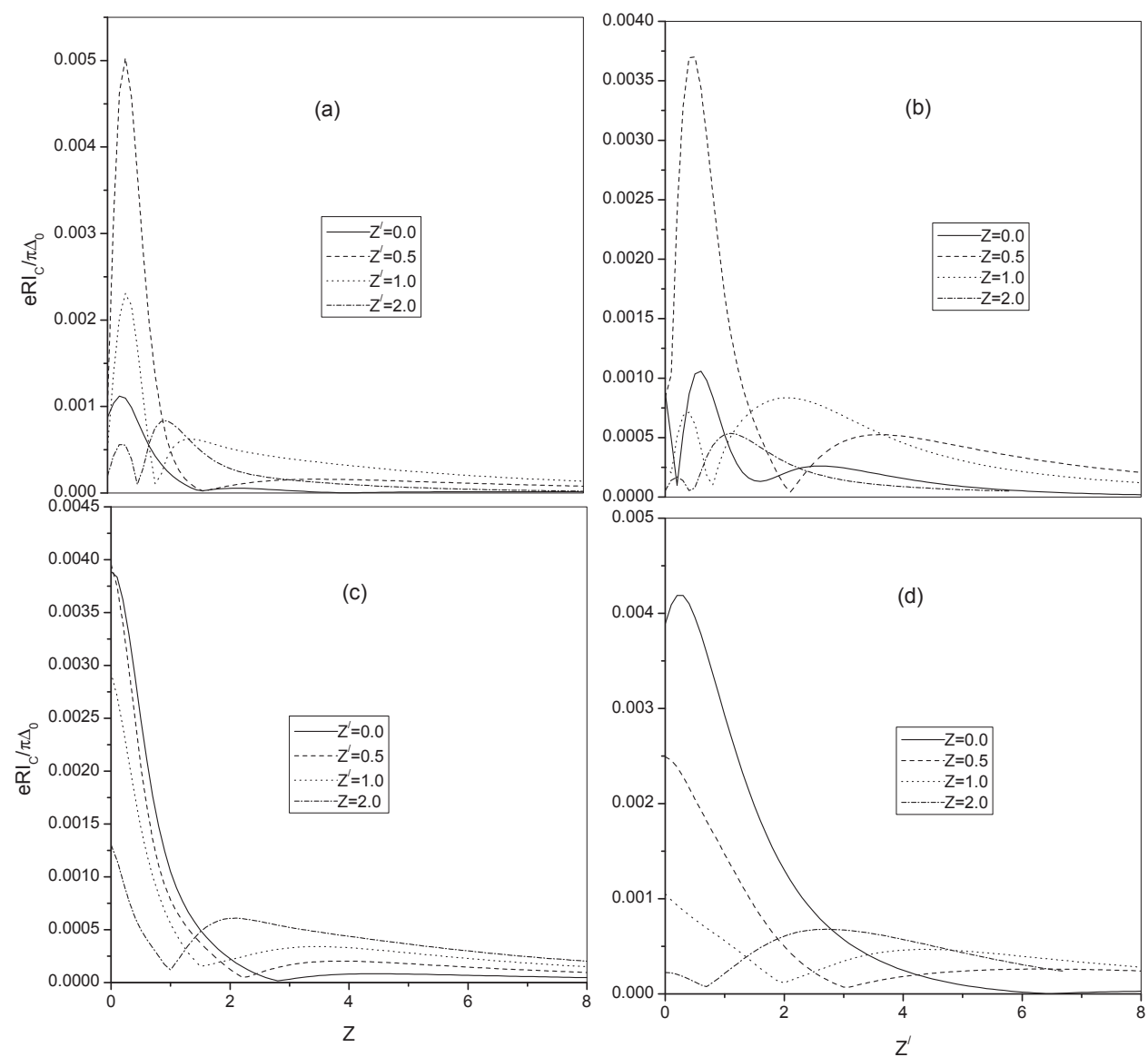

Fig. 8. Dependence of $I_{C}$ on $Z$ and $Z^{\prime}$ at $P=0.9$ with the same situations as in Fig. 6 (from Ref. 35). 

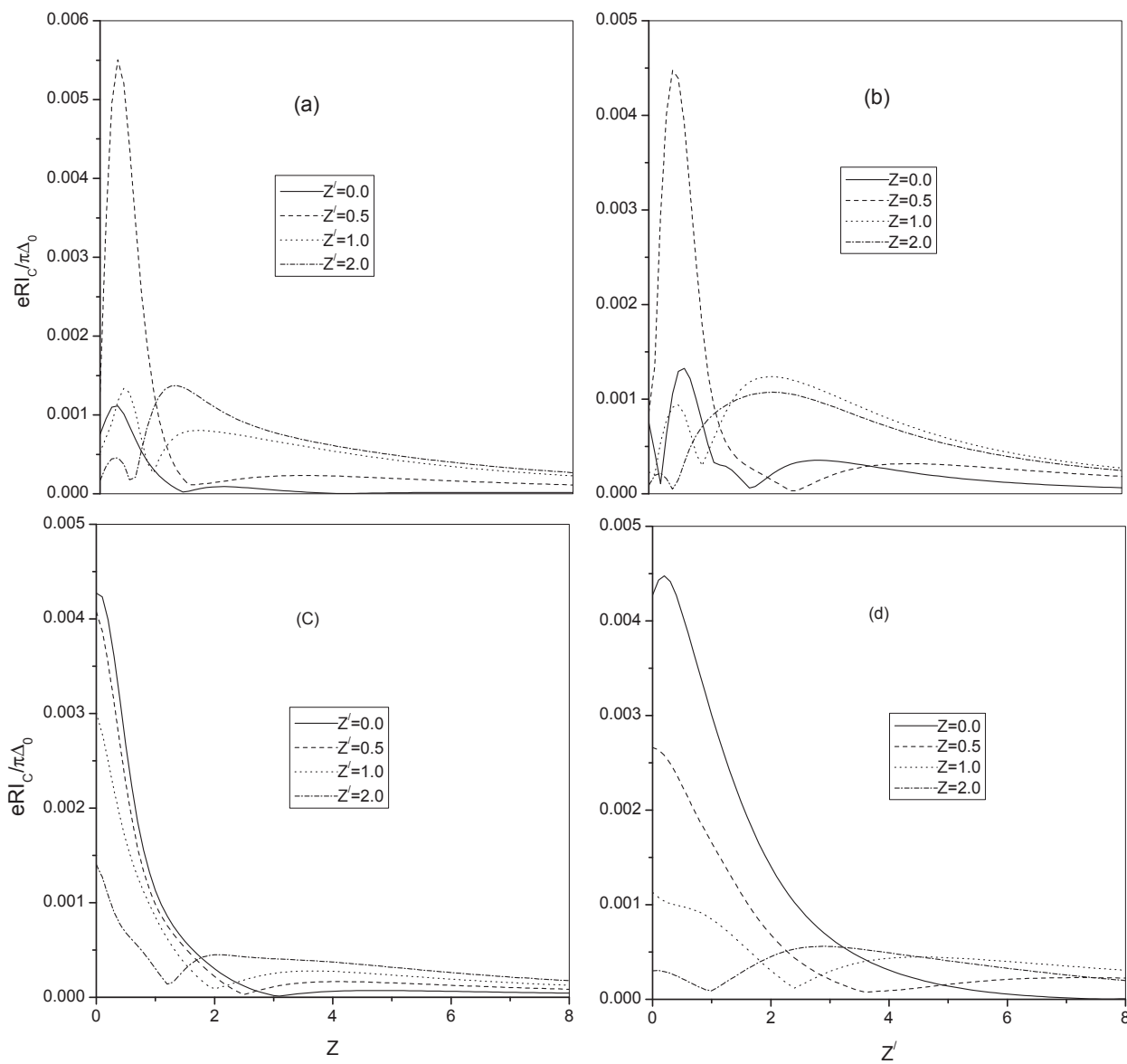

Fig. 9. Dependence of $I_{C}$ on $Z$ and $Z^{\prime}$ at $P=0.9$ with the same situations as in Fig. 7 (from Ref. 35). 


\section{Symbol list}

SC $\cdots$ superconductor

FS $\cdots$ ferromagnetic semiconductor

SM $\cdots$ semiconductor

BDG … Bogoliubov-de Gennes (BDG) equation

ELQ …electronlike quasiparticle

HLQ . . holelike quasiparticle

$n \cdots$ n-type semiconductor

$p \cdots$ p-type semiconductor

$\phi \cdots$ a phase difference between the SCs

$I \cdots$ the dc Josephson current

$I_{C} \cdots$ the critical Josephson current

$V(x) / V(\boldsymbol{r}) \cdots$ the usual static potential

$U \cdots$ the barrier height

$\delta(x) \cdots \delta$-type function

$H_{F S}(\boldsymbol{r}) \cdots$ the hole Hamiltonian in the FS

$H_{0}(\boldsymbol{r}) \cdots$ the hole Hamiltonian of the SM GaAs

$\hbar$... Planck constant

$m_{p} / m_{e} \cdots$ the mass of a hole/electron

$h(r) \cdots$ the effective exchange field

$\sigma \cdots$ the conventional Pauli spin operator

$\Gamma \cdots$ the spin splitting energy

$J_{p d} \cdots$ the $\mathbf{p}-\mathrm{d}$ exchange coupling strength

$<S^{z}>\cdots$ the thermal average of the impurity Mn ion spins

$p \cdots$ the relative spin-polarized degree

$E_{F} \cdots$ the fermi-energy

$\zeta_{k} \cdots$ the excitation energy

$\epsilon_{k} \cdots$ the one-electron energy

$\Delta_{0} \cdots$ energy gap at zero temperature

$\Delta \cdots$ energy gap at non-zero temperature

$\Delta(x) \cdots$ the pair potential 
$u_{\sigma}, v_{\bar{\sigma}} \cdots$ the two-component wavefunction

$\psi(x) \cdots$ the wave function

$a, b, c, d \cdots$ transmission and reflection coefficients

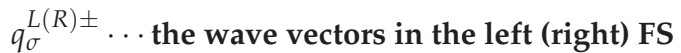

$q^{\prime \pm} \ldots$ the wave vectors in the SM

$k^{ \pm} \ldots$ the wave vectors in the SCs

$k_{\|} \cdots$ the parallel component of the wave vector

$k_{F} \cdots$ the Fermi wave vector

$Z / Z^{\prime} \cdots$ two dimensionless parameters

$k_{B} \cdots$ boltzmann factor

$T \cdot$ tempreture

$\omega \cdots$ the Matsubara frequencies

\section{References}

[1] B. M. Andersen. Y. S. Barash, S. Graser, and P.J. Hirschfeld, Phys. Rev. B 77, 054501 (2008).

[2] I. Zǔtic, J. Fabian, and S. Das Sarma, Rev. Mod. Phys. 76, 323 (2004).

[3] A.I. Buzdin, Rev. Mod. Phys. 77, 935 (2005)

[4] T. Jungwirth, J. Sinova, J. Mašek, J. Kučera, and A.H. MacDonald, Rev. Mod. Phys. 78, 809 (2006); H. Saito, S. Yuasa, and K. Ando, Phys. Rev. Lett 95, 086604 (2005).

[5] H. Ohno, A. Shen. F. Matsukura, A. Oiwa, A. Endo, S. Katsumoto, and Y. Iye, Appl. Phys. Lett 69, 363 (1996); H. Ohno, D. Chiba, F. Matsukura, T. Omiya, E. Abe, T. Dietal, Y. Ohno, and K. Ohtani, Nature (London) 408, 994 (2000); K.C. Ku, S.J. Potashnik, R.F. Wang, S.H. Chun, P. Schiffer, N. Samarth, M.J. Seong, A. Mascarenhas, E. Johnston-Halperin, R.C. Myers, A.C. Gossard, and D.D. Awschalom, Appl. Phys. Lett. 82,2302 (2003).

[6] H. Ohno, Science 281, (1991); J. Magn. Magn. Matter. 200, 110 (1999); T. Dietl, H. Ohno, F. Matsukura, J. Cibert, and D. Ferrand, Science 287, 019 (2000); J. Konig, H.-H. Lin, and A.H. MacDonald, Phys. Rev. Lett. 84, 5682 (2000); J. Konig, T. Jungwirth, and A.H. MacDonald, Phys. Rev. B 64, 184423 (2001).

[7] Y.C. Tao, J.G. Hu, and H. Liu, J. Appl. Phys. 96, 498 (2004)

[8] Y.C. Tao and J.G. Hu, Int. J. Mod. Phys B 16, 2247 (2004).

[9] B.D. Josephson, Phys. Lett. 1, 251 (1962).

[10] V.V. Ryazanov, V.A. Oboznov, A. Yu. Rusanov, A.V. Veretennikov, A.A. Golubov, and J. Aarts, Phys. Rev. Lett. 86, 2427 (2001).

[11] V.V. Ryazanov, V.A. Oboznov, A.V. Veretennikov, A. Yu. Rusanov Phys. Rev. B. 65, 020501 (2001).

[12] T. Kontos, M. Aprili, J. Lesueur, and X. Grison, Phys. Rev. Lett 86, 304 (2001).

[13] J.M.E. Geers, M.B.S. Hesselberth, J. Aarts, and A.A.Golubov, Phys. Rev. B 64, 094506 (2001). 
[14] O. Bourgeois, P. Gandit, J. Lesueur, A. Sulpice, X. Grison, and J. Chaussy, Eur, Phys. J. B 21, 75 (2001).

[15] T. Kontos, M. Aprili, J. Lesueur, F. Genetêt, B. Stephanidis, and R. Boursier, Phys. Rev. Lett. 89, 137007 (2002).

[16] M. Fogelström, Phys. Rev. B 62, 11812 (2000).

[17] Yu. S. Barash and I. V. Bobkova, Phys. Rev. B 65, 144502 (2002).

[18] F.S. Bergeret, A.F. Volkov, and K.B. Efetov, Phys. Rev. B 64, 134506 (2001).

[19] V.N. Krivoruchko and E. A. Koshina, Phys. Rev. B 64, 172511 (2001).

[20] N.M. Chtchelkatchev, W. Belzig, Yu. V. Nazarov, and C. Bruder, Pis'ma Zh. Éksp. Teor. Fiz. 74, 357 (2001) [JEPT Lett. 74, 323 (2001)].

[21] A.A. Golubov, M. Yu. Kupriyanov, and Ya. V. Fominov, Pis'ma Zh. Éksp. Teor. Fiz. 75, 709 (2002) [JEPT Lett. 75, 588 (2002)].

[22] Yu. S. Barash, I. V. Bobkova, and T. Kopp, Phys. Rev. B 66, 140503 (2003).

[23] A. Yu. Zyuzin, B. Spivak, and M. Hruška, Europhys. Lett. 62, 97 (2003).

[24] Z. Radović, L. Dobrosavljević-Grujić, and B. Vujičić, Phys. Rev. B 63, 214512 (2001); M. Božović and Z. Radović, Phys. Rev. B 66, 134524 (2002); Z. Radović, N. Lazarides, and N. Flytzanis, Phys. Rev. B 68, 014501 (2001).

[25] K. Halterman and O.T. Valls, Phys. Rev. B 65, 014509 (2002); M. Zareyan, W. Belzig, and Yu. V. Nazarov, Phys. Rev. Lett. 86, 308 (2001).

[26] A. Brinkman and A.A. Golubov, Phys. Rev. B 61, 11297 (2000).

[27] I. Baladie and A. Buzdin, Phys. Rev. B 64, 224514 (2001).

[28] F.S. Bergeret, A.F. Volkov, and K.B. Efetov, Phys. Rev. B 65, 134505 ( 2002).

[29] J.S. Jang, D. Davidović, D.H. Reih, and C.L. Chien, Phys. Rev. Lett. 74, 314 (1995); Y. Obi, M. Ikebe, T. Kubo, and H. Fujimori, Physica C 317-318, 149 (1999).

[30] D.J. van Harlingen, Rev. Mod. Phys. 67, 515 (1995).

[31] J.X. Zhu, B. Friedman and C.S. Ting, Phys. Rev. B 59, 9558 (1999).

[32] J.X. Zhu and C.S. Ting, and A.V. Balatsky Phys. Rev. B 66,

[33] C.R. Hu, Phys. Rev. Lett. 72, 1526 (1994).

[34] Y.C. Tao, J. Appl. Phys. 100, 063901 (2006)

[35] Y.C. Tao, Eur. Phys. J. B. 56, 83 (2007).

[36] P.G. de Gennes, Superconductivity of Metal and Alloys (Benjamin, New York, 1996).

[37] A. Furusaki and M. Tsukada, Solid State Commun. 78, 299 (1991).

[38] W.M. van Huffelen, T.M. Klapwijk, D.R. Heslinga, M.J. de Boer, and N. van der Post, Phys. Rev. B 47, 5170-5189 (1993).

[39] M.J. Storcz and F.K. Wilhelm, Appl. Phys. Lett. 83, 2589 (2003). 


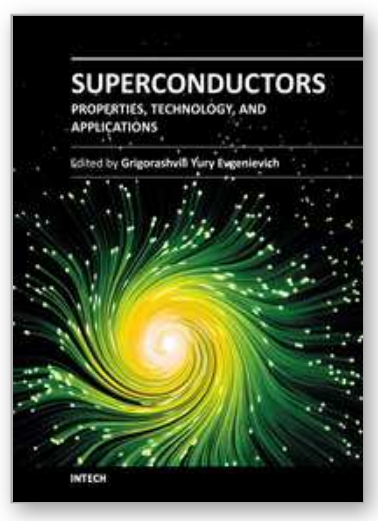

\author{
Superconductors - Properties, Technology, and Applications \\ Edited by Dr. Yury Grigorashvili
}

ISBN 978-953-51-0545-9

Hard cover, 436 pages

Publisher InTech

Published online 20, April, 2012

Published in print edition April, 2012

Book "Superconductors - Properties, Technology, and Applications" gives an overview of major problems encountered in this field of study. Most of the material presented in this book is the result of authors' own research that has been carried out over a long period of time. A number of chapters thoroughly describe the fundamental electrical and structural properties of the superconductors as well as the methods researching those properties. The sourcebook comprehensively covers the advanced techniques and concepts of superconductivity. It's intended for a wide range of readers.

\title{
How to reference
}

In order to correctly reference this scholarly work, feel free to copy and paste the following:

Y.C. Tao (2012). Josephson Current in a Ferromagnetic Semiconductor/Semiconductor/Ferromagnetic Semiconductor Junction with Superconducting Contacts, Superconductors - Properties, Technology, and Applications, Dr. Yury Grigorashvili (Ed.), ISBN: 978-953-51-0545-9, InTech, Available from: http://www.intechopen.com/books/superconductors-properties-technology-and-applications/josephson-currentin-a-ferromagnetic-semiconductor-semiconductor-ferromagnetic-semiconductor-junctio

\section{INTECH}

open science | open minds

\section{InTech Europe}

University Campus STeP Ri

Slavka Krautzeka 83/A

51000 Rijeka, Croatia

Phone: +385 (51) 770447

Fax: +385 (51) 686166

www.intechopen.com

\section{InTech China}

Unit 405, Office Block, Hotel Equatorial Shanghai

No.65, Yan An Road (West), Shanghai, 200040, China 中国上海市延安西路65号上海国际贵都大饭店办公楼 405 单元

Phone: +86-21-62489820

Fax: +86-21-62489821 
(C) 2012 The Author(s). Licensee IntechOpen. This is an open access article distributed under the terms of the Creative Commons Attribution 3.0 License, which permits unrestricted use, distribution, and reproduction in any medium, provided the original work is properly cited. 